\title{
ON RELATIVELY FREE SUBSETS OF LIE GROUPS
}

\author{
BERNARD R. GELBAUM
}

\begin{abstract}
In an arbitrary neighborhood $U$ of the identity $e$ of a connected Lie group there is a subset $S$ of cardinality c and relatively free, i.e., the only nontrivial equations $x_{1}^{\varepsilon_{1}} x_{2}^{\varepsilon_{2}} \cdots x_{n}^{\varepsilon_{n}}=e, \varepsilon_{i}= \pm 1$, satisfied by substitution for distinct symbols among the $x_{i}$ distinct elements of $S$ are equations that are identities throughout $G$.
\end{abstract}

0 . In [2] the existence of the free topological group $F(X)$ associated with a completely regular space $X$ is shown by a construction involving quaternions. In brief (and corrected) form, the argument proceeds as follows: the (algebraic) free group $F_{0}(X)$ generated by $X$ is embedded isomorphically in the group $\mathbf{H}_{1 \infty}(X) \equiv P_{f} \mathbf{H}_{1 f}$, the Cartesian product of the multiplicative group $\mathbf{H}_{1}$ of quaternions of norm 1 (each $\mathbf{H}_{1 f}=\mathbf{H}_{1}$ ) where the index $f$ ranges over $C\left(X, \mathbf{H}_{1}\right)$, the set of continuous maps $f: X \rightarrow \mathbf{H}_{1}$. In this embedding, $X$ preserves its topology and $F_{0}(X)$ is endowed with the topology of a topological group. Standard results show that $\sigma_{\max }=\sup \left\{\mathcal{T}: F_{0}(X)\right.$ is a topological group in the topology $\mathcal{T}$ and $X$ inherits its topology from $\mathcal{T}$ \} $\equiv \sup \left\{T G F_{0}(X)\right\}$ produces $F(X)$. The role played by the compact group $\mathbf{H}_{1 \infty}(X)$ is that of insuring that the set $T G F_{0}(X)$ is nonempty.

The argument hinges on the existence of an infinite free subset of $\mathbf{H}_{1}$. Since $\mathbf{H}_{1}$ is a connected Lie group, a question related to this aspect of $\mathbf{H}_{1}$ is explored below and is answered as follows: In every connected Lie group $G$ there is a subset $S$, of cardinality $\mathrm{c} \equiv \operatorname{card}(\mathbf{R})$ and as free as any subset of $G$ can be: if a word evaluated on $S$ yields $e$ (the identity of $G$ ), then the word is identically $e$ on $G$ (see below for details). In particular, if no (word) identities hold universally in $G$, then $S$ is free.

1. The proof depends on two lemmas, the second of which emerged in the form given below as a result of illuminating conversations with Professor S. Schanuel of SUNY/Buffalo. The first lemma is given in [1], [4]. The proof below is somewhat more elementary than that in [4] and is therefore included here.

LEMMA 1. Let $f\left(x_{1}, x_{2}, \ldots, x_{n}\right)$ be a real-or complex-valued function of the real variables $x_{1}, x_{2}, \ldots, x_{n}$. If, for some constant a, the $n$-dimensional measure $\mu_{n}\left(f^{-1}(a)\right)$ is positive, then $f \equiv a$ in any region $R$ where $f$ is analytic and such that $R \supset f^{-1}(a)$.

Received by the editors November 14, 1974 and, in revised form, March 3, 1975.

AMS (MOS) subject classifications (1970). Primary 22E15, 22E10, 22E05, 20F05, 20F 15.

Key words and phrases. Free topological groups, Lie groups and (relatively) free subsets thereof. 
Proof. We may assume that for some cube $C=\left\{\left(x_{1}, x_{2}, \ldots, x_{n}\right):\left|x_{i}-b_{i}\right|\right.$ $\left.\leqslant \varepsilon_{i}>0, i=1,2, \ldots, n\right\}$ contained in $R, \mu_{n}\left(C \cap f^{-1}(a)\right)>0$ and that in $C$

$$
f\left(x_{1}, \ldots, x_{n}\right)=\sum_{0<k_{i}} a_{k_{1} \cdots k_{n}}\left(x_{1}-b_{1}\right)^{k_{1}} \cdots\left(x_{n}-b_{n}\right)^{k_{n}}
$$

converges uniformly and absolutely. Thus we may write

$$
f\left(x_{1}, \ldots, x_{n}\right)=\sum_{0<k_{1}} f_{1 k_{1}}\left(x_{2}, \ldots, x_{n}\right)\left(x_{1}-b_{1}\right)^{k_{1}}
$$

where $f_{1 k_{1}}$ are analytic functions of $x_{2}, \ldots, x_{n}$. The Fubini theorem shows that for each $\left(x_{2}, \ldots, x_{n}\right)$ in some set $S_{n-1}$ of positive $(n-1)$-dimensional measure, $\mu_{1}\left\{x_{1}:\left(x_{1}, x_{2}, \ldots, x_{n}\right) \in C \cap f^{-1}(a)\right\}>0$. The set of such $x_{1}$ has a nonempty derived set in $\mathbf{C}$ and so $f_{1 k_{1}}\left(x_{2}, \ldots, x_{n}\right) \equiv 0, k_{1} \geqslant 1$, $f_{10}\left(x_{2}, \ldots, x_{n}\right)=a$ on $S_{n-1}$.

Since the lemma obtains (in even stronger form!) for $n=1$, an argument by induction completes the proof.

Lemma 2. (S. Schanuel) Let $\psi: M \rightarrow N$ be an analytic map between analytic manifolds $M$ and $N$. Let $K=\{x: x \in M, \psi$ is constant in some neighborhood of $x$ \}. Then $K$ is closed (and clearly open).

Proof. Let $p_{0} \in \bar{K} \equiv$ closure of $K$ in $M$. Then there are points $p_{n} \in K$, $n \geqslant 1, p_{n} \rightarrow p_{0}$, and for each $n \geqslant 1, \psi$ is constant on some neighborhood $V_{n}$ of $p_{n}$. Let $B_{M}$ be a cubical (open) neighborhood in $\mathbf{R}^{\operatorname{dim} M}$ and let $f_{M}: B_{M} \rightarrow M$ provide a chart around $p_{0}$; let $B_{N}, f_{N}: B_{N} \rightarrow N$ serve similarly for $\psi\left(p_{0}\right)$. Then if the coordinate maps for an $f^{-1}$ are denoted by $f_{i}^{-1}$ we find

$$
h_{i} \equiv f_{N i}^{-1} \circ \psi \circ f_{M}: B_{M} \rightarrow \mathbf{R}, \quad i=1,2, \ldots, \operatorname{dim} M,
$$

are analytic maps of $B_{M}$ into $\mathbf{R}$ and for all $n \geqslant$ some $n_{1}$, each is constant on the open subset $f_{M}^{-1}\left(f_{M}\left(B_{M}\right) \cap V_{n}\right)$ of $B_{M}$. Since $\mu\left(f_{M}^{-1}\left(f_{M}\left(B_{M}\right) \cap V_{n}\right)\right)>0$, Lemma 1 shows that each $h_{i}$ is constant on $B_{M}$. Hence $\psi \circ f_{M}$ is constant on $B_{M}, \psi$ is constant on $f_{M}\left(B_{M}\right)$, an open set containing $p_{0}$. Hence $p_{0} \in K$ and $K$ is closed.

Corollary. If $M$ is connected, $K=\varnothing$ or $K=M$, i.e., a nonconstant analytic map of a connected manifold cannot be locally constant.

REMARK. There is a clear connection between the results above and those in [1]. The author is obliged to the referee for the citation.

2. We make some clarifying remarks about words and groups. A word $W_{l k}$ is defined by a finite sequence of $l$ distinct symbols $u_{1}, u_{2}, \ldots, u_{l}$, a finite sequence of signs $\varepsilon_{1}, \varepsilon_{2}, \ldots, \varepsilon_{k}= \pm 1$, where $k \geqslant l$ and a "product" $w_{1}^{\varepsilon_{1}} \cdots w_{k}^{\varepsilon_{k}}$ where each $w_{i}$ is some $u_{j}$. It is assumed that in $w_{1}^{\varepsilon_{1}} \cdots w_{k}^{\varepsilon_{k}}$ there is no finite sequence $w_{p}, w_{p+1}, \ldots, w_{p+q}$ and some symbol $u_{j}$ such that $w_{p}$ $=w_{p+1}=\cdots=w_{p+q}=u_{j}$ and $\sum_{i=p}^{p+q} \varepsilon_{i}=0$. Such situations are inessential and will be excluded from discussion. Thus we deal only with essential words.

For a group $G$ and a subset $A \subset G$, the notation $A^{\times l}$ signifies the $l$-fold Cartesian product of $A$ with itself, as distinguished from $A^{l}$, the set $\left\{a_{1} a_{2} \cdots a_{l}: a_{i} \in A, i=1,2, \ldots, l\right\}$. For a word $W_{l k}$ as described there is defined on $G^{\times l}$ a function $\tilde{W}_{l k}: G^{\times l} \rightarrow G$ given by 


\section{the result of replacing in the "product" $\tilde{W}_{l k}\left(g_{1}, g_{2}, \ldots, g_{l}\right)=w_{1}^{\varepsilon_{1}} \ldots w_{k}^{\varepsilon_{k}}$ each $u_{i}$ by $g_{i}, i=1$, the resulting element of $G$.}

A word $W_{l k}$ is nontrivial for the group $G$ if $\tilde{W}_{l k} \neq e$ on $G \times l$, where $e$ is the identity of $G$.

In $G^{\times l}$ there is the "antidiagonal" $E_{l}=\left\{\left(g_{1}, g_{2}, \ldots, g_{l}\right): g_{p} \neq g_{q}\right.$ for $1 \leqslant p \neq q \leqslant l\}$. A subset $S$ of $G$ is called relatively free if for every word $W_{l k}$ nontrivial for $G, \tilde{W}_{l k} \neq e$ throughout $S^{\times l} \cap E_{l}, l \leqslant k=2,3, \cdots$. If every (essential) word $W_{l k}$ is nontrivial for $G$, then a relatively free set as just defined is free in the usual sense.

THeOREM 1. Let $G$ be a connected Lie group of dimension $d \geqslant 1$. Then for every neighborhood $U$ of the identity $e$ in $G$, there is in $U$ a relatively free set $S$ such that $\operatorname{card}(S)=\operatorname{card}(\mathbf{R}) \equiv \mathfrak{c}$.

Proof. Let $\left(B_{2}, f\right)$ provide a chart for a neighborhood contained in $U$ and assume $f(0)=e ; f^{-1}: f\left(B_{2}\right) \rightarrow B_{2}$ gives rise to $d$ coordinate maps $f_{i}^{-1}: f\left(B_{2}\right)$ $\rightarrow \mathbf{R}$. We assume $B_{2}=\left\{\left(x_{1}, \ldots, x_{d}\right):\left|x_{i}\right|<2\right\}$ and we set $\bar{B}_{1}$ $=\left\{\left(x_{1}, \ldots, x_{d}\right):\left|x_{i}\right| \leqslant 1\right\}$. Finally, let $D$ be the countable Cartesian product of $\bar{B}_{1}$ with itself. We may assume Lebesgue measure $\mu_{d}$ is normalized in $\mathbf{R}^{d}$ so that $\mu_{d}\left(\bar{B}_{1}\right)=1$; thereby we may introduce product measure $\mu$ in $D$, where, in particular $\mu(D)=1$. An element of $D$ is a sequence $\left\{d_{\nu}\right\}^{\infty}$ of $d$-tuples of real numbers. A sequence $\delta \equiv\left\{d_{\nu}\right\} \in D$ is called binding iff, for some word $W_{l k}$ nontrivial for $G$ and at some point in $\left(\left\{f\left(d_{\nu}\right)\right\}_{1}^{\infty}\right)^{\times l} \cap E_{l} \equiv f(\delta ; l), \tilde{W}_{l k}=e$. In the preceding, $\left\{f\left(d_{\nu}\right)\right\}_{1}^{\infty}$ is the set $f\left(d_{1}\right), f\left(d_{2}\right), \ldots$ in $G$.

Note that for each $k \in \mathbf{N}$ there are only finitely many words $W_{l k}$ hence finitely many words nontrivial for $G$. For each word $W_{l k}$ the set of values of $\tilde{W}_{l k}$ on $f(\delta, l)$ is countable. Furthermore, $\tilde{W}_{l k}=e$ iff $f_{i}^{-1} \circ \tilde{W}_{l k}=0, i=1$, $2, \ldots, d$.

If $W_{l k}$ is nontrivial for $G$, then $\tilde{W}_{l k} \neq e$ on $G^{\times l}$. Since the map $\tilde{W}_{l k}$ : $G^{\times l} \rightarrow G$ is analytic and since $G$ and thus also $G^{\times l}$ are connected, Lemma 2 applies and shows that there is no neighborhood on which $\tilde{W}_{l k}$ is constant. In particular, $\tilde{W}_{l k}$ cannot reduce to $e$ throughout $f\left(B_{1}\right)^{\times l}$.

Hence for any word $W_{l k}$ nontrivial for $G$, the analytic functions

$$
f_{i}^{-1} \circ \tilde{W}_{l k}(\underbrace{f, f, \ldots, f)}_{l \text {-terms }}: B_{1}^{\times l} \rightarrow \mathbf{R}
$$

are not all constant. Thus, by Lemma 1 ,

$$
\underbrace{\mu_{d} \times \mu_{d} \times \cdots \times \mu_{d}}_{l \text {-factors }}\left\{\left(d_{1}, d_{2}, \ldots, d_{l}\right):\right. \text {. }
$$

Thus for each set of $l$ distinct indices $\nu_{1}, \nu_{2}, \ldots, \nu_{l}$ and for each word $W_{l k}$ nontrivial for $G$,

$$
\mu\left\{\delta \equiv\left\{d_{\nu}\right\}: \tilde{W}_{l k}\left(f\left(d_{\nu_{1}}\right), f\left(d_{\nu_{2}}\right), \ldots, f\left(d_{\nu_{l}}\right)\right)=e\right\}=0 .
$$

Hence, for $W_{l k}$ fixed, 


$$
\begin{aligned}
& \mu\left\{\delta \equiv\left\{d_{\nu}\right\}: \tilde{W}_{l k}\left(f\left(d_{\nu_{1}}\right), f\left(d_{\nu_{2}}\right), \ldots, f\left(d_{\nu_{l}}\right)\right)=e\right. \\
&\left.\quad \text { for some set of } l \text { distinct indices } \nu_{1}, \nu_{2}, \ldots, \nu_{l}\right\}=0 .
\end{aligned}
$$

Hence, if

$$
\beta=\left\{\delta \equiv\left\{d_{\nu}\right\}: \tilde{W}_{l k}\left(f\left(d_{\nu_{1}}\right), \ldots, f\left(d_{\nu_{l}}\right)\right)=e \text { for some word } W_{l k}\right.
$$

and some set of $l$ distinct indices $\left.\nu_{1}, \ldots, \nu_{l}\right\}$,

then clearly $\beta$ contains the set of binding sequences and $\mu(\beta)=0$. In particular $D \backslash \beta \neq \varnothing$. Hence, if $\left\{d_{\nu}\right\} \in D \backslash \beta$, then $\left\{d_{\nu}\right\}$ is not binding. The set $S_{0} \equiv\left\{f\left(d_{\nu}\right)\right\}_{1}^{\infty}$ leads to the set $S$ promised, as the following lines show.

First note that $\nu_{1} \neq \nu_{2}$, then $f\left(d_{\nu_{1}}\right) \neq f\left(d_{\nu_{2}}\right)$. Otherwise for the clearly nontrivial word $W_{22} \equiv u_{1} u_{2}^{-1}$, the substitution $u_{1} \rightarrow f\left(d_{\nu_{1}}\right), u_{2} \rightarrow f\left(d_{\nu_{2}}\right)$ shows that $\left\{d_{v}\right\} \in \beta$, a contradiction. Thus $S_{0}$ is infinite.

Second, $S_{0}$ is relatively free since if $\widetilde{W}_{l k}=e$ at some point of $S_{0}^{\times l} \cap E_{l}$ and if $W_{l k}$ is nontrivial for $G$, then $\left\{d_{\nu}\right\}$ is binding.

Clearly card $\left(S_{0}\right)=\kappa_{0} \equiv \operatorname{card}(\mathbf{N})$. An argument in [3] may be modified as follows to show that there is a relatively free set $S_{\max } \subset U$ and $\operatorname{card}\left(S_{\max }\right)$ $=c$. In [3] the following lemma is proved: If $\mathscr{F} \equiv\left\{F_{\alpha}\left(x_{1}, \ldots, x_{n}\right)\right\}$ is $a$ family of functions analytic on an open cube $C^{0}=\left\{\left(x_{1}, x_{2}, \ldots, x_{n}\right):\left|x_{i}-a_{i}\right|\right.$ $\left.<\varepsilon_{i}>0, i=1,2, \ldots, n\right\}$, if no $F_{\alpha} \equiv 0$ and if $\operatorname{card}(\mathscr{F})<\mathfrak{c}$, then there is an $n$-tuple $\left(b_{1}, b_{2}, \ldots, b_{n}\right)$ such that, for all $\alpha, F_{\alpha}\left(b_{1}, \ldots, b_{n}\right) \neq 0$. In [3] a transfinite induction establishes the existence of a free subset of cardinality $c$ in the rotation group $O(n, \mathbf{R})$ in Euclidean space $\mathbf{R}^{n}, n \geqslant 3$. Used in that development are (a) the existence in $O(3, \mathbf{R})$ of a free set of two elements (this fact was proved by R. Robinson [6] in the context of F. Hausdorff's result [5] on rotations in $\mathbf{R}^{3}: O(3, \mathbf{R}) \supset F / N, F$ the free group on two generators $a, b$, and $N$ the normal subgroup generated by $a^{2}$ and $b^{3}$; Robinson showed that in $F$ the free group generated by $a b a b$ and $a b^{2} a b^{2}$ meets $N$ in the identity; thus $O(3, \mathbf{R})$ contains a free group on two generators); (b) Kurosh's theorem showing that a free group contains an infinite free set. In both [3] and [5], as in the present discussion, use is made of the theory of analytic functions of several real variables.

The following lines are in essence a Zorn's-lemma version of the final argument in [3]. In the nonempty set IRF of infinite relatively free subsets of $U$ there is, via Zorn's lemma applied to IRF partially ordered by inclusion, a maximal set $S$. We show $\gamma \equiv \operatorname{card}(S)=$ c. Clearly $\gamma \leqslant c$. If $\gamma<\mathfrak{c}$, for each word $W_{l k}$ nontrivial for $G$, each substitution, whereby $l-1$ symbols are replaced by fixed and distinct elements $f\left(d_{1}\right), \ldots, f\left(d_{l-1}\right)$ of $S$ and the other symbol is replaced by an arbitrary $y \in f\left(B_{2}\right)$, yields a function $\tilde{W}_{l k}^{y}$ that depends analytically on $y$, i.e., the functions

$$
k_{i}(y) \equiv f_{i}^{-1} \circ \tilde{W}_{l k}^{y} \circ(f, f, \ldots, f)\left(d_{1}, d_{2}, \ldots, d_{l-1}, y\right)
$$

are analytic maps of $B_{2}$ into $\mathbf{R}$. For at least one $i, k_{i}(y)$ is not identically 0 in $B_{2}$ since if $y$ is in $S$, but $y \notin\left(f\left(d_{1}\right), f\left(d_{2}\right), \ldots, f\left(d_{l-1}\right)\right)$, then $\tilde{W}_{l k}^{y} \neq e$. Here the fact that $S$ is infinite is used in an essential way.

The cardinality of the set of all such functions (derived from the (countable) set of all words nontrivial for $G$, the set of all one-to-one substitutions of 
the kind described (here the cardinality is not more than $\gamma^{\prime}=\gamma$ ) and all $i=1,2, \ldots, d$ as needed) has cardinality $<c$. Thus by the lemma of [3] as quoted above, this set of functions has a common nonzero: $b_{1}^{0}, b_{2}^{0}, \ldots, b_{n}^{0}$. If $y_{0}=f\left(b_{1}^{0}, \ldots, b_{n}^{0}\right)$ and if $g$ is any element of the group generated by $S$, then $y_{0} \neq g$ since otherwise $y_{0}^{-1} g$ is simultaneously some $、 \tilde{W}_{l k}^{y_{0}}$ and also $e$, a contradiction. Thus, in particular $y_{0} \notin S$ and $S \cup\left\{y_{0}\right\} \supsetneqq S$ is a relatively free set since every $\tilde{W}_{l k}^{y_{0}} \neq e$. The maximality of $S$ is contradicted, whence $\gamma=\mathfrak{c}$, and $S$ is the set promised in the theorem.

It should be noted, in view of Paul Cohen's results and the subsequent developments of several others working in set theory, that the de GrootDekker lemma is a trivial consequence of Lemma 1 of the present paper if one accepts the continuum hypothesis as an axiom.

Indeed, the family $\mathscr{F}$ is countable since card $(\mathscr{F})<c$ and the continuum hypothesis is an axiom. Thus the union $N$ of the sets $N_{\alpha}$ of zeros of the $F_{\alpha}$ is, by Lemma 1, the countable union of null sets, hence $N$ is itself a null set. Hence $C^{0} \backslash N$ is nonempty and any $\left(b_{1}, b_{2}, \ldots, b_{n}\right)$ in $C^{0} \backslash N$ satisfies: $F_{\alpha}\left(b_{1}, b_{2}, \ldots, b_{n}\right) \neq 0$, all $\alpha$.

Variations on this theme when the continuum hypothesis is denied are also relevant; e.g., there are set-theory models in which the continuum hypothesis is false but in which Lebesgue measure is "c-additive", i.e., if $\left\{N_{\alpha}\right\}$ is a family of null sets and $\operatorname{card}\{\alpha\}<c$ then $\cup_{\alpha} N_{\alpha}$ is a null set. Thus, in the notation above, $N=\cup_{\alpha} N_{\alpha}$ is a null set even if $\{\alpha\}$ is not countable, but card $\{\alpha\}<c$. The argument just given then shows $F_{\alpha} \neq 0$ on $C^{0} \backslash N$.

In another direction there is a characterization of the words that may serve as identities for a Lie group $G, \operatorname{dim} G \geqslant 1$. If $\tilde{W}_{l k} \equiv e$ on $G^{\times l}$, then $\Sigma_{w_{i}=u_{j}} \varepsilon_{i}=0, j=1,2, \ldots, l$. Indeed, otherwise, for some $p \in \mathbf{N}, g^{p} \equiv e$ for all $g$ in $G$. Hence for any one parameter subgroup $\{g(t):-\infty<t<\infty\}$ $\equiv H_{g}, g(p t) \equiv e$ for all $t$. Thus any tangent vector, at $e$ to $H_{g}$, is 0 , in contradiction of a basic property of Lie groups.

On the other hand, if $\sum_{w_{i}=u_{j}} \varepsilon_{i}=0, j=1,2, \ldots, l$, then every Abelian group $A$, whether it is a Lie group or not, is one for which $\tilde{W}_{l k} \equiv e$ on $A^{\times l}$. We conclude: $a$ word $W_{l k}$ is admissible as a universal restriction on a Lie group $G$, i.e., $\tilde{W}_{l k} \equiv e$ on $G^{\times l}$, iff $\sum_{w_{i}=u_{j}} \varepsilon_{i}=0, j=1,2, \ldots, l$.

\section{BIBLIOGRAPHY}

1. J. E. Coury, On the measure of zeros of coordinate functions, Proc. Amer. Math. Soc. 25 (1970), 16-20. MR 41 \#395.

2. B. R. Gelbaum, Free topological groups, Proc. Amer. Math. Soc. 12 (1961), 737-743. MR 25 \#4025.

3. J. de Groot and T. Dekker, Free subgroups of the orthogonal group, Compositio Math. 12 (1954), 134-136. MR 16, 672.

4. R. Gunning and H. Rossi, Analytic functions of several complex variables, Prentice-Hall, Englewood Cliffs, N.J., 1965. MR 31 \#4927.

5. F. Hausdorff, Grundzüge der Mengenlehre, Veit, Leipzig, 1914; photographic reproduction, Chelsea, New York, 1949. MR 11, 88.

6. R. M. Robinson, On the decomposition of spheres, Fund. Math. 34 (1947), 246-260. MR 10, 106.

Department of Mathematics, SUNy at Buffalo, Buffalo, New York 14226 